\title{
Satisfaction, Anxiety, Depression and Stress among Family Members of Patients in Malaysian Intensive Care Unit
}

\author{
Mohd Ariffudin Abdul Hamid, Mohd Basri Mat Nor
}

Department of Anaesthesiology and Intensive Care, Kulliyyah of Medicine, International Islamic University Malaysia

Introduction: Family satisfaction has been identified as a quality indicator in critical care area. The impact of family satisfaction level was also found to be associated with symptoms of psychological distress such as anxiety, depression and stress among family members. We evaluated the satisfaction level and prevalence, risk factor and correlation of psychological distress symptoms with the satisfaction level among family members in Malaysian Intensive Care Unit (ICU). Materials and Method: This is a cross-sectional, multicentre study conducted in ICU at Hospital Sultanah Aminah Johor Bahru and International Islamic University Medical Centre. Family members were enrolled 3 days after ICU admission, and they completed a modified version of Critical Care Family Needs Inventory (CCFNI) and the Depression, Anxiety and Stress Scales (DASS). Results: A total of 176 family members were enrolled in this study. We found $116(66 \%)$ of the family members scores $\geq 3$ denoting satisfaction with the mean CCFNI score was 3.11 (SD=0.3). Prevalence of depression, anxiety and stress were of $30.1 \%, 41.4 \%$ and $28.9 \%$ respectively. Risk factor for psychological distress symptoms were female sex, spousal relationship, lower education, median income less than RM 4000, staying with patient and younger patient age. Negative correlation between depression, anxiety and stress with CCFNI score were found $(p<0.05)$ but with low correlation coefficient $(r=-0.178$ to -0.209$)$. Family members without symptoms of psychological distress were more satisfied (higher CCFNI score) with ICU care compared to those with symptoms of psychological distress $(p<0.05)$. Conclusion: Family members of ICU patient were satisfied with the care provided in the ICU. High rates of psychological distress symptoms in this study and its correlation with the satisfaction level highlight the need to identify and implement preventive and management strategies for family members to improve the overall ICU care. 